Results In RA synovial tissue expression of FoxO1 negatively correlated with clinical parameters of disease activity: serum C-reactive protein $(R=-0.771, P=0.0008)$, erythrocyte sedimentation rate $(R=-0.739, P=0.0003)$, and DAS28 $(R=-0.575, P=0.01)$, as well as synovial IL-6 mRNA levels $(R=-0.628, P=0.004)$. In vitro, RA FLS stimulation with IL-1 $\beta$ or TNF $\alpha$ caused rapid downregulation of FoxO1 mRNA levels, followed by reduction of FoxO1 protein expression and DNA binding. This effect was independent of PKB signalling, and was associated with acceleration of FoxO1 mRNA degradation in the presence of IL-1 $\beta$. Inhibition of c-Jun N-terminal kinase (JNK), but not other MAPKs, prevented downregulation of FoxO1 expression and binding by IL-1 $\beta$, and blocked IL-1 $\beta$-induced reduction of FoxO1 mRNA stability. Overexpression of constitutively active FoxO1 in RA FLS induced apoptosis associated with altered expression of genes regulating cell cycle and apoptosis: BIM and p27 $7^{\mathrm{kip} 1}$ were induced while expression of Bcl-XL was suppressed in cells expressing active FoxO1.

Conclusions Collectively, our findings suggest that suppressed synovial FoxO1 expression is strongly associated with RA pathology and demonstrate that reduction of FoxO1 expression might contribute to perpetuation of inflammation in RA by promoting FLS survival and proliferation. Our data also identify JNK-mediated modulation of FoxO1 mRNA stability as an important mechanism underlying regulation of FoxO1 by inflammatory cytokines.

\section{A10.17 INTERLEUKIN-36 $\alpha$ IS EXPRESSED BY SYNOVIAL PLASMA CELLS AND INDUCES CYTOKINE PRODUCTION IN HUMAN FIBROBLASTS}

doi:10.1136/annrheumdis-2013-203224.17

${ }^{1}$ Silke Frey, ${ }^{1}$ Anja Derer, 'Maria-Elena Messbacher, ${ }^{2}$ Serena Bugatti, ${ }^{3} \mathrm{D}$ Baeten, ${ }^{4}$ Carlomaurizio Montecucco, 'Georg Schett, 'Axel J Hueber. 'Department of Internal Medicine 3 and Institute for Clinical Immunology, University hospital of Erlangen, Erlangen, Germany; 'Division of Rheumatology, University of Pavia School of Medicine, IRCCS Policlinico San Matteo Foundation, Pavia, Italy; ${ }^{3}$ Academic Medical Center, University of Amsterdam, Amsterdam, Netherlands; " University of Pavia School of Medicine, IRCCS Policlinico San Matteo Foundation, Pavia, Italy

Background and Objectives The interleukin (IL)-36 $\alpha$ is a recently described member of the IL-1 cytokine family with pro-inflammatory and clearly pathogenic properties in psoriasis arthritis (PsA). The majority of patients with PsA and rheumatoid arthritis (RA) benefit from cytokine blocking therapies against TNF $\alpha$; however, despite novel developments, subgroups of patients do not respond to this therapy. Therefore it is necessary to get a better understanding of the pathogenesis of synovitis in PsA and RA to learn more about the complex cellular interplay and to develop new treatment approaches. Therefore, we wanted to determine the IL-36 $\alpha$ expression in PsA compared to RA and osteoarthritis (OA).

Materials and Methods Synovial tissue obtained from arthritis patients were stained for IL-36 $\alpha$, IL-36 receptor (IL-36R) and IL-36R antagonist (IL-36Ra) by immunohistochemistry and immunofluorescence. Lysates were tested for IL-36 $\alpha$ by immunoblotting. Synovial fibroblasts (FLS) cultured in the presence of IL-36 $\alpha$ were assessed for cytokine expression by quantitative real time PCR and Multiplex assay. IL-36 $\alpha$-induced signal transduction in FLS was analysed by immunoblotting.

Results The IL-36R and its ligands IL-36 $\alpha$ and IL-36Ra could be detected in inflammatory arthritis in the synovial lining layer as well as cellular infiltrates. IL-36 $\alpha$ was significantly higher expressed in PsA and RA synovium compared to OA ( $p=0.0011$ and $p<0.0001$, respectively). No differences were seen in IL-36R and IL-36Ra. The expression of IL-36 $\alpha$ was confirmed by western blot analysis. IL-36 $\alpha$ induced expression of IL-6 and IL-8 in FLS. CD138-positive plasma cells were defined as a major cellular source for IL-36 $\alpha$. Functionally, IL-36 $\alpha$ induced the expression of IL- 6 and IL-8 in FLS through NF- $\kappa B /$ p38-activation.
Conclusions Here, we describe that the novel cytokine IL-36 $\alpha$, mainly expressed by plasma cells, is upregulated in PsA and RA synovium and leads to IL- 6 and IL-8 production by synovial fibroblasts. This finding needs further studies to determine if the IL-36 family can function as a potential target for arthritis therapy.

\section{A10.18 LACK OF ASSOCIATION OF SERUM INTERLEUKIN-17 AND INTERLEUKIN-23 LEVELS WITH DISEASE ACTIVITY IN PATIENTS WITH ANKYLOSING SPONDYLITIS IN LATVIA}

doi:10.1136/annrheumdis-2013-203224.18

1,2Zepa Julija, ${ }^{1,5}$ Bulina Inita, ${ }^{3}$ Nikitina-Zake Liene, ${ }^{3}$ Klovins Janis, ${ }^{1,5}$ Jurka Anita, ${ }^{1,2}$ Tretjakovs Peteris, ${ }^{2,4}$ Lejnieks Aivars, ${ }^{1,5}$ Andersone Daina. 'Pauls Stradins Clinical University Hospital; ${ }^{2}$ Riga Stradins University; ${ }^{3}$ Biomedical Research and Study Centre of Latvia; ${ }^{4}$ Riga East Clinical University hospital; ${ }^{5}$ University of Latvia, Latvia

Background Ankylosing spondylitis (AS) is a clinically wellknown chronic inflammatory disease of the axial skeleton and peripheral joints. The pathogenesis of this disease still remains a challenge. Determination of cytokine profile and its role involved in AS pathogenesis give an opportunity to extend the targeted therapeutic approach. Interleukin-17 (IL-17) and interleukin-23 (IL-23) are cytokines of interest in the investigation of the pathogenesis of spondyloarthritides although their importance in AS is not clearly defined.

Objectives to investigate levels of IL-17 and IL-23 in a group of AS and in a demographically matched group of healthy subjects and its association with the disease activity measured by relevant clinical and biochemical parameters.

Materials and Methods 39 AS patients classified by the modified New York and ASAS criteria were assessed clinically and $6 \mathrm{ml}$ of serum were collected from each patient. 39 healthy subjects as control group were included in this study. The serum IL-17 and IL-23 levels were tested using xMAP multiplex immunobead assay technology. At the same time the disease activity was measured by using Bath Ankylosing Spondylitis Disease Activity Index (BASDAI) and Ankylosing Spondylitis Disease Activity Score (ASDAS) using C-reactive protein (CRP), erythrocyte sedimentation rate (ESR).

Results The mean serum IL-17 and IL-23 level in AS group was respectively 18.9 (SD 39.6) and 194.6 (SD 261.4) pg/ml. In the healthy control group the mean serum IL-17 level was 15.4 (SD 26.0) and IL-23 level - 200.3 (SD 256.3) pg/ml. The serum levels of IL-17 and IL-23 were not statistically significantly different from the healthy subjects and the levels did not correlate with the disease activity measured by BASDAI and ASDAS (using the CRP and ESR). Conclusions These results suggest that IL-17 and IL-23 are not major components of the pathogenesis of inflammation in AS patients. Our data differ from Chen W S et al, in 2012 published data of the serum IL-17 and IL-23 level association with the disease activity in Chinese patients with AS. This difference is probably due to the various genetic aspects characterising AS as geographically matched disease.

\section{A10.19 MRP8/14 SERUM COMPLEXES AS PREDICTOR OF RESPONSE TO BIOLOGICAL TREATMENTS IN RHEUMATOID ARTHRITIS}

doi:10.1136/annrheumdis-2013-203224.19

'Yuen Kei Choi, 'Marieke J Herenius, 'Carla A Wijbrandts, 'Johannes Roth, ${ }^{2}$ Dirk Föll, 'Danielle M Gerlag, ${ }^{1,3}$ Paul-Peter Tak, ${ }^{2,4}$ Dirk Holzinger. 'Division of Clinical Immunology and Rheumatology, Academic Medical Center/University of Amsterdam, The Netherlands; 'Institute of Immunology, University Hospital Muenster, Muenster, Germany; ${ }^{3}$ GlaxoSmithKline, Stevenage, UK; ${ }^{4}$ Department of General Paediatrics, University Children's Hospital Muenster, Muenster, Germany

Background and Objectives Biological therapy has dramatically improved the treatment of rheumatoid arthritis (RA). One-third of 